\title{
The Right Journal, Editor, and Referees, at the Right Time
}

After being in the $\mathrm{PhD}$ world for more than a decade, I can honestly say that I do not understand very well the logic of publication based on rigor and originality. This contradiction started to build up in my early scholar years when one of my first papers was finally published in a reputed journal after having been rejected in 4 other low-ranked journals in our field. When I was talking about this paradox with one of my mentors, Anthony Leicht, he perfectly summarized the point in this sentence: "It is about the right journal, and the right editor, with the right referees, at the right time." Thus, in this editorial, I explain what the word "right" would mean in each case for IJSPP.

The "right" journal refers to the IJSPP's scope and mission. However, sometimes, this is not sufficient to clearly identify what our journal wants to publish. If you are not a frequent reader of $I J S P P$, I recommend you read recent issues and pay attention to the articles related to your specific topic. This process may help you switch onto the same frequency at which we are communicating sport science. While the topics we tend to publish are easy to identify, I consider the practical applications the most important section of any manuscript. ${ }^{1}$ A good practical application should address and solve real problems of competitive athletes, from recreational to elite levels, with less effort. However, studies confirming negative or trivial results on current practices are also welcome.

The "right" editor refers to the associate editor (AE) who receives the manuscript after a first assessment by the editor, who may discuss the suitability of manuscripts with the AE. A good cover letter may be helpful to highlight the merits of your work at this stage. Whether your manuscript goes into peer review or receives a "desk or administrative rejection" mainly depends on the rigor of your methods and the impact, pertinence, and originality of your proposal. Fortunately, IJSPP has an excellent team of AEs with quite different backgrounds and expertise within the sport science community, ranging from specialists to generalists (including myself). This facilitates the work by the editor, who needs to look for the "right" AE. However, the availability of a specific $\mathrm{AE}$ for your submission is not always guaranteed. In any case, you can trust that your manuscript will be handled honestly by a wellprepared $\mathrm{AE}$ and that desk rejections are always based on fast but fair evaluations. We are handling the work of other colleagues, from students to professors, and this is a serious responsibility for us.

The "right" time would refer to how well your manuscript competes with the quantity and quality of submissions and recent publications at that time. For example, because of the large number of submissions during the 2020 lockdown, some good manuscripts that might have easily gone into peer review in another time were finally desk rejected. This is why I personally prefer to submit my manuscripts during holidays, when the submission rates are lower in all journals. If you receive a desk rejection, you may formally question the decision by the editors, but please refer to the merits of your manuscript, and not your record of publications. We editors are not the owners of the truth, ${ }^{2}$ but we always try to do our best to follow objective criteria.

The concept of peer review is nearly as old as the history of scientific publication. The selection of the "right" reviewers is probably the most controversial aspect of this process. We are a small scientific community, and there are often professional and nonprofessional issues potentially affecting a manuscript's evaluation. In addition, some specific topics have an extremely limited number of true experts around the world. For these reasons, please honestly indicate your suggested and opposed reviewers to assist with this process. The AEs will try to find the most suitable reviewers, but we all know that they are busy most of the time. To manage this problem, AEs can have a more proactive role while handling the manuscripts, including their own participation as a reviewer. If you are invited to review, please try to help us publish the best sport science. ${ }^{3}$ Sometimes, we receive potentially good papers by authors not as experienced as you. Helping others is, for me, the most valuable aspect of peer review. If you feel peer review is a waste of time, or simply cannot meet the requirements of a fair peer review on time, simply decline the invitation, but try to recommend a suitable alternative. Do know, though, that being a reviewer has some rewards and impacts not always explicitly recognized, including the improvement of your own scientific skills and the opportunity to influence the work of others.

Finally, whether your manuscript is accepted or rejected, consider that this is not the end. Nobel laureates, including Hans Krebs, have received rejections on some of the top-cited papers of all time. ${ }^{4}$ On the other hand, it is a fact that most accepted papers receive few or no citations at all. ${ }^{5}$ This is the best part of our business: Science is self-correcting, ${ }^{6}$ and only Chronos can judge the real impact of your accepted manuscript. Therefore, remember the inherent randomness associated with this process (see Figure 1), and do not call in vain the family of the $\mathrm{AE}$ who signed the rejection letter. Just accept the received feedback and keep trying.

Daniel Boullosa, IJSPP Associate Editor, Federal University of Mato Grosso do Sul, Brazil

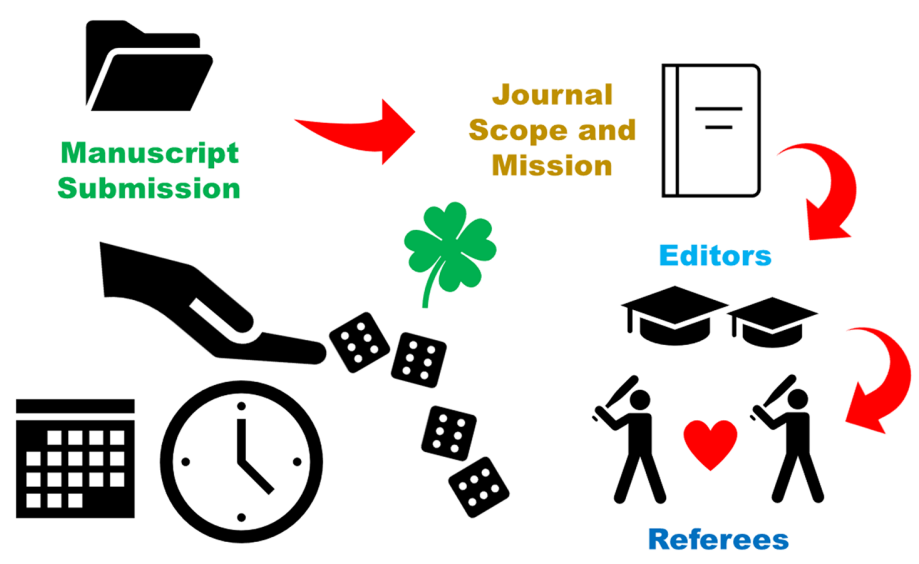

Figure 1 - Diagram flux of a manuscript's submission. Note that some degree of randomness is always present at any stage of this process. 


\section{References}

1. Coutts AJ. Building a bridge between research and practice-the importance of the practical application. Int J Sports Physiol Perform. 2020;15(4):449. PubMed ID: 32182588 doi:10.1123/ijspp.2020-0143

2. Foster C, Anholm JD, Best T, et al. Scientific discovery and its role in sports science. Kinesiology. 2016;48:274-284.

3. Chamari K, Beneke R. The game is fair play: a kind reminder to scientists about their role in keeping science running. Int $J$ Sports Physiol Perform. 2017;12(9):1125-1126. PubMed ID: 29214916 doi:10.1123/ijspp.2017-0615
4. Campanario JM, Acedo E. Rejecting highly cited papers: the views of scientists who encounter resistance to their discoveries from other scientists. J Am Soc Inf Sci Technol. 2007;58(5):734-743. doi:10. 1002/asi.20556

5. Meho LI. The rise and rise of citation analysis. Phys World. 2006;20(1):32-36. doi:10.1088/2058-7058/20/1/33

6. Foster C. Sport science: progress, hubris, and humility. Int J Sports Physiol Perform. 2019;14(2):141-143. PubMed ID: 30663915 doi:10.1123/ijspp.2018-0982 